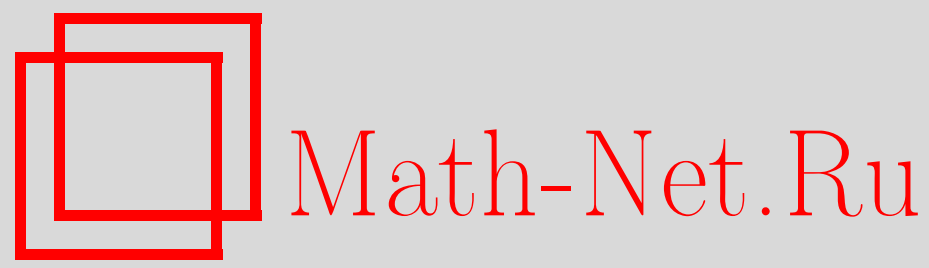

В. И. Бердышев, Аппроксимация обобщенными полиномами, обеспечивающая наилучшую привязку, Матем. заметки, 1996, том 60, выпуск 5, 658-669

DOI: https://doi.org/10.4213/mzm1879

Использование Общероссийского математического портала MathNet.Ru подразумевает, что вы прочитали и согласны с пользовательским соглашением

http://www. mathnet.ru/rus/agreement

Параметры загрузки:

IP : 54.224 .60 .19

26 апреля 2023 г., $14: 46: 38$

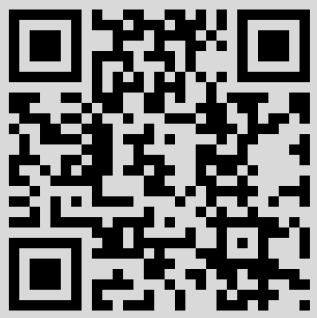


том 60 выпуск 5 нояБрь 1996

\section{АППРОКСИМАЦИЯ ОБОБЩЕННЫМИ ПОЛИНОМАМИ, ОБЕСПЕЧИВАЮЩАЯ НАИЛУЧШУЮ ПРИВЯЗКУ}

\section{В.И. Бердышев}

Введение. Далее $f$ - непрерывная на компакте $Q \subset \mathbb{R}^{N}$ функция, $\Delta$ компакт из $\mathbb{R}^{N}, 0 \in \Delta$. Пусть $t \in \mathbb{R}^{N}, \Delta_{t}:=\Delta+t \subset Q$. Функцию

$$
\varphi(x)=\varphi_{t}(x)=f(x+t) \quad(x \in \Delta)
$$

будем называть фрагментом функции $f$ (соответствующим точке $t$ ). В приложениях встречается такая задача, когда известны функции $f(x)$ $(x \in Q), \varphi(x)(x \in \Delta)$, известно, что $\varphi$ является фрагментом функции $f$, и требуется найти местоположение фрагмента, т.е. найти $t$, для которого выполняется условие (1). Приведем пример (см. [1], [2]): автономно управляемьй аппарат двигается над регионом $Q ; f(x)$ - значение некоторой геофизической характеристики (высоты, оптической яркости и т.д.) в точках $x \in Q$; в бортовом компьютере аппарата хранятся значения $f(x)$ для всех $x \in Q$; находясь над точкой $t \in Q$, координаты которой неизвестны или известны с погрешностью, апшарат снимает фрагмент (1) $\varphi=\varphi(x)(x \in \Delta)$ функции $f$; требуется определить местоположение $t$, используя информацию о $f$ и $\varphi$. Случай, когда по техническим условиям имеется возможность замерять значения поля $f$ лиш в конечном числе точек, также охватьвается приведенной постановкой, он соответствует частному случаю, когда $\Delta-$ сетка из $\mathbb{R}^{N}$.

Задачу определения $t$ по известным $f=f(x)(\forall x \in Q)$ и $\varphi=\varphi(x)$ $(\forall x \in \Delta)$ будем назьвать задачей привязки (навигачии). В [3], [4] рассмотрен случай функций одной переменной.

Задача привязки не корректна по Адамару, как задача обращения отображения $t \mapsto \varphi=\varphi_{t}$, не являющегося, вообще говоря, взаимно однозначным. В п. 1 данной работы изучается свойство информативности функ-

Работа выполнена при поддержке Российского фонда фундаментальных исследований, грант № 93-011-196. 
ции $f$, т.е. свойство, обеспечивающее возможность привязки по любому ее фрагменту.

Часто при решении практических задач налагается жесткое ограничение на объем памяти бортового компьютера, выделяемой для хранения информации о функции $f$. В таких случаях приходится вместо $f$ хранить аппроксимирующую функцию $p$, которая задается небольшим числом параметров, и осушествлять привязку посредством $p$ и фрагмента $\varphi$. Это может привести к увеличению ошибки привязки. В п. 2 рассматривается задача определения в заданном классе $\mathscr{P}$ аппроксимируюшей функции $p$, привязка по которой с помошью фрагмента $\varphi$ функции $f$ осуществляется с наименьшей ошибкой. Приводятся необходимые условия на такую функцию.

1. Модуль информативности функции. В качестве решения задачи привязки по функции $f$ и фрагменту $\varphi$ естественно взять [1], [2] одно из значений $T=T(t)$, реализующих нижнюю грань

$$
\inf \left\{\left\|f(x+T)-\varphi_{t}(x)\right\|_{\Delta}: \Delta_{T} \subset Q\right\},
$$

где $\|\cdot\|_{\Delta}$ - некоторая норма, например, $\|f\|_{\Delta}=\max \{|f(x)|: x \in \Delta\}$. Евклидово расстояние $|t-T(t)|$ между точками $t, T(t) \in \mathbb{R}^{N}$ естественно назвать ошибкой привязки. В идеальном случае нижняя грань (2) равняется нулю. Однако на практике, даже если функция $f$ задана с приемлемой погрешностью и фрагмент $\varphi$ снимается достаточно точно, применение конкретного алгоритма минимизации по $T \in \mathbb{R}^{N}$ позволяет получить малую, но не нулевую величину $\|f(x+T)-\varphi(x)\|_{\Delta}$. Важным с точки зрения привязки является свойство информативности функции $f$, гарантируюшее малость величины $|t-T|$ при малой $\left\|f(T+x)-\varphi_{t}(x)\right\|_{\Delta}$. Это свойство характеризуется посредством функции [3]:

$$
J_{\Delta}(\tau, f)=\sup _{Y, T}\left\{|Y|: \Delta_{T} \subset Q,\|f(x)-f(x+Y)\|_{\Delta_{T}} \leqslant \tau\right\},
$$

которую будем называть модулем информативности. Обозначим через $I_{\Delta}$ класс функций $f$, для которых $J_{\Delta}(\tau, f) \rightarrow 0(\tau \rightarrow 0)$. Непосредственно из определения $J_{\Delta}$ следует оценка погрешности привязки

$$
|t-T| \leqslant J_{\Delta}\left(\left\|f(x+T)-\varphi_{t}(x)\right\|_{\Delta}, f\right)
$$

если при практическом вычислении (2) найдено $T$, для которого величина $\left\|f(x+t)-\varphi_{t}(x)\right\|_{\Delta}$ достаточно мала, то при $f \in I_{\Delta}$ неравенство (3) влечет малость ошибки $|t-T|$. Через модуль информативности можно оценить 
погрешность привязки и в том случае, когда привязка осушествляется по фрагменту (1) функции $f \in I_{\Delta}$ и по функции $p$, аппроксимирующей функцию $f$, т.е. когда вместо $(2)$ решается задача

$$
d(p, t):=\inf _{T}\left\{\left\|p(x+T)-\varphi_{t}(x)\right\|_{\Delta}: \Delta_{T} \subset Q\right\}
$$

Эта оценка имеет вид [3], [4]

$$
|t-T| \leqslant J_{\Delta}\left(2\|f-p\|_{Q}, f\right)
$$

Ввиду (3), (5) представляет интерес вопрос о поведении модуля $J_{\Delta}(\tau, f)$ в нуле. Отметим, что если $f$-строго монотонная на $[a, b]$ функция, а $\Delta=\{0\}$, то $J(\tau, f)=\omega\left(\tau, f^{-1}\right)-$ модуль непрерьвности [5] обратной функции. Приведем несколько случаев, когда $J_{\Delta}(\tau, f)$ можно оценить сверху. Далее $\|f\|_{D}=\max \{|f(x)|: x \in D\}, D=Q, \Delta$.

1) Пусть $Q=[a, b] \subset \mathbb{R}, \Delta=[0, \delta], 0<\delta<b-a, f=\sum_{k=0}^{n} a_{k} x^{k}$, $a_{n} \neq 0$, тогда $[3],[4]$

$$
J_{\Delta}(\tau, f) \leqslant \frac{\tau}{\left|a_{n}\right| n E_{n-1}}
$$

где

$$
E_{k}=\inf \left\{\left\|x^{k}+\alpha_{k-1} x^{k-1}+\alpha_{k-2} x^{k-2}+\cdots+\alpha_{0}\right\|: \alpha_{k-1}, \ldots, \alpha_{0} \in \mathbb{R}\right\}
$$

и по теореме П. Л. Чебьшева $E_{k}=(\delta / 2)^{k} / 2^{k-1}$. Здесь отрезок $Q$ может быть неограниченньг.

ПРЕДЛОЖЕНИЕ 1 (А.В. Нятин). Ecлu $f_{n m}=p_{n}(x) / q_{m}(x) \neq$ const, $p_{n}(x)=\sum_{k=0}^{n} a_{k} x^{k}, a_{n} \neq 0, q_{m}(x)=\sum_{k=0}^{m} b_{k} x^{k}>0$ на $[a, b]\left(b_{m}=1\right)$, mo

$$
J_{\Delta}\left(\tau, f_{n m}\right) \leqslant \begin{cases}\frac{\left\|q_{m}\right\|_{[a, b]}^{2}}{\left|a_{n}\right| \cdot|n-m| E_{n+m-1}} & (n \neq m), \\ \frac{\left\|q_{n}\right\|_{[a, b]}^{2} \tau}{\left|a_{n-1}-a_{n} b_{n-1}\right| E_{2 n-2}} & (n=m) .\end{cases}
$$

В самом деле, при $n \neq m$

$$
\begin{aligned}
\left\|\frac{p_{n}(x+Y)}{q_{m}(x+Y)}-\frac{p_{n}(x)}{q_{m}(x)}\right\|_{\Delta_{T}} & =\left\|\frac{a_{n}(n-m) x^{n+m-1}+\cdots}{q_{m}(x) q_{m}(x+Y)}\right\|_{\Delta_{T}} \\
& \geqslant \frac{\left|a_{n}\right| \cdot|n-m| E_{n+m-1}}{\left\|q_{m}\right\|_{[a, b]}^{2}}
\end{aligned}
$$


а случай $n=m$ сводится к случаю $n \neq m$ следующим образом:

$$
\begin{aligned}
& \frac{a_{n} x^{n}+\cdots+a_{0}}{x^{n}+b_{n-1} x^{n-1}+\cdots+b_{0}} \\
& =a_{n}+\frac{\left(a_{n-1}-a_{n} b_{n-1}\right) x^{n-1}+\cdots+\left(a_{0}-a_{n} b_{0}\right)}{x^{n}+b_{n-1} x^{n-1}+\cdots+b_{0}} .
\end{aligned}
$$

2) Рассмотрим случай многочленов двух переменных степени $n$

$$
p=p_{n}(x, y)=\sum_{k+l \leqslant n} a_{k l} x^{k} y^{l},
$$

заданных на квадрате $Q=[-1,1] \times[-1,1], \Delta=[0, \delta] \times[0, \delta](0<\delta<2)$. Для $T=(X, Y) \in \mathbb{R}^{2}$ имеем

$$
p(x+X, y+Y)-p(x, y)=\sum_{k=0}^{n-1} c_{k} x^{n-k-1} y^{k}-p_{n-2},
$$

где

$c_{k}=c_{k}(T)=(n-k) a_{n-k, k} X+(k+1) a_{n-k-1, k+1} Y \quad(k=0,1, \ldots, n-1)$,

a $p_{n-2}=p_{n-2}(x, y)-$ многочлен степени не более $n-2$. Обозначим

$$
\begin{gathered}
E(p, T)_{D}=\min _{p_{n-2}}\left\|\sum_{k=0}^{n-1} c_{k} x^{n-k-1} y^{k}-p_{n-2}\right\|_{D} \quad(D \subset Q), \\
E(p)=\min _{|T|=1} E(p, T)_{Q} .
\end{gathered}
$$

Замена переменных дает

$$
E(p, T)_{Q}=\left(\frac{2}{\delta}\right)^{n-1} E(p, T)_{\Delta}
$$

Поскольку коэффициенты $c_{k}$ из формулы (7) зависят от $X, Y$ линейно, то $\min \left\{E(p, T)_{Q}:|T|=\lambda\right\}=\lambda E(p)(\lambda>0)$, далее,

$$
\min _{\|T\|=\lambda} E(p, T)_{\Delta}=\left(\frac{\delta}{2}\right)^{n-1} \lambda E(p),
$$

и значит (см. (6)), для $|T|=\lambda$

$$
\|p(x+X, y+Y)-p(x, y)\|_{\Delta} \geqslant\left(\frac{\delta}{2}\right)^{n-1} \lambda E(p) .
$$

Из (8) следует 
ПРЕДЛОЖЕНИЕ 2. Если многочлен $p=p_{n}(x, y)$ таков, что ни при каком $T,|T|=1$, коэффициенты $c_{k}(7)$ не обращаются в нуль одновременно, то $E(p)>0 u$

$$
J_{\Delta}(\tau, p) \leqslant\left(\frac{2}{\delta}\right)^{n-1} \frac{\tau}{E(p)} .
$$

Для оценки величины $E(p)$ можно воспользоваться неравенством А. А. Маркова (см., например, [6])

$$
\begin{gathered}
\left\|\sum_{k=0}^{n} b_{k} x^{k}\right\|_{[-1,1]} \geqslant A_{n k}\left|b_{k}\right| \quad(k=0, \ldots, n), \\
\frac{k !}{2^{k-1} n}\left(\frac{n-k}{2}\right) !\left[\left(\frac{n+k}{2}-1\right) !\right]^{-1}, \quad n-k \text { четно, } \\
\frac{k !}{2^{k-1}(n-1)}\left(\frac{n-k-1}{2}\right) !\left[\left(\frac{n+k-3}{2}\right) !\right]^{-1}, n-k \text { нечетно. }
\end{gathered}
$$

Полагая в $p(x, y) \quad y=\alpha x+\beta(|\alpha| \leqslant 1,|\beta| \leqslant 1)$, получим

$$
\begin{aligned}
\sum_{k=0}^{n} \sum_{i+j=k} a_{i j} x^{i} y^{j} & =\sum_{k=0}^{n} \sum_{j=0}^{k} a_{k-j, j} x^{k-j} y^{j} \\
& =\sum_{k=0}^{n} \sum_{j=0}^{k} \sum_{l=0}^{j} a_{k-j, j} c_{j}^{l} \alpha^{j-l} \beta^{l} x^{k-l} \\
& =\sum_{m=0}^{n} \sum_{l=0}^{n-m} \sum_{j=l}^{m+l} a_{m+l-j, j} c_{j}^{l} \alpha^{j-l} \beta^{l} x^{m} \\
& =\sum_{m=0}^{n} x^{m} \sum_{l=0}^{n-m} \beta^{l} \sum_{j=l}^{m+l} a_{m+l-j, j} c_{j}^{l} \alpha^{j-l},
\end{aligned}
$$

где $c_{k}^{l}=k ! /((k-l) ! l !)$. Трижды применим неравенство Маркова по переменным $x, \beta$ и $\alpha$ последовательно, тогда

$$
\left\|\sum_{i+j \leqslant n} a_{i, j} x^{i} y^{j}\right\|_{C[-1,1]} \geqslant A_{n, m} A_{n-m, l} A_{m, j-l} c_{j}^{l}\left|a_{m+l-j, j}\right|
$$

для любых $m=0, \ldots, n, l=0, \ldots, n-m, j=l, \ldots, m+l$. Полагая $x=\gamma y+\delta(|\gamma| \leqslant 1,|\delta| \leqslant 1)$ и рассуждая аналогично, будем иметь

$$
\left\|\sum_{i+j \leqslant n} a_{i j} x^{i} y^{j}\right\|_{C[-1,1]} \geqslant A_{n, m} A_{n-m, l} A_{m, i-l} c_{i}^{l}\left|a_{i, m+l-i}\right|
$$

для любых $m=0, \ldots, n, l=0, \ldots, n-m, i=l, \ldots, m+l$. Таким образом, установлено 
ПРЕДЛОЖЕНИЕ 3. Справедливо неравенство

$$
\begin{aligned}
& \left\|\sum_{k+l \leqslant n} a_{k l} x^{k} y^{l}\right\|_{[-1,1]} \\
& \quad \geqslant A_{n, m} A_{n-m, l} A_{m, j-l} c_{j}^{l} \max \left\{\left|a_{m+l-j, j}\right|,\left|a_{j, m+l-j}\right|\right\}
\end{aligned}
$$

(см. (9)) для любых $m=0, \ldots, n, l=0, \ldots, n-m, j=l, \ldots, m+l$.

ЗАмЕЧАниЕ 1 . В случае $n=2$ величина $E(p)$ может быть найдена в явном виде:

$$
\begin{aligned}
E\left(p_{2}\right) & =\min _{|X|,|Y|=1} \min _{c} \max _{|x|,|y| \leqslant 1}\left|\left(2 a_{20} X+a_{11} Y\right) x+\left(a_{11} X+2 a_{02} Y\right) y+c\right| \\
& =\min _{|X|,|Y|=1}\left(\left|2 a_{20} X+a_{11} Y\right|+\left|a_{11} X+2 a_{02} Y\right|\right)=\frac{\left|a_{11}^{2}-4 a_{20} a_{02}\right|}{\max \left\{\left|a_{20}\right|,\left|a_{02}\right|\right\}}
\end{aligned}
$$

и, значит, при $a_{11}^{2} \neq 4 a_{20} a_{02}$

$$
J_{\Delta}\left(\tau, p_{2}\right) \leqslant \frac{2 \max \left\{\left|a_{20}\right|,\left|a_{02}\right|\right\}}{\left|a_{11}^{2}-4 a_{20} a_{02}\right| \delta} \tau .
$$

\section{2. Аппроксимация, наилучшая с точки зрения привязки.} Пусть компакт $Q \subset \mathbb{R}^{N}$ вьпукльй, $f$ непрерьвна на $Q, \varphi=\varphi_{t}(x)$ - фрагмент функции $f$, соответствуюший точке $t \in \mathbb{R}^{N} ;$ пусть также задан класс $\mathscr{P}(\mathscr{P} \subset C(Q))$ аппроксимируюших функций (например, класс многочленов степени не более $n)$. В дальнейшем требуется лишь, чтоб $\mathscr{P}$ был линеен, а функции $p \in \mathscr{P}$ были бы непрерывно дифференцируемы на $Q$.

Дадим определение функции $p \in \mathscr{P}$ наилучшей привязки [3]. Для $p \in \mathscr{P}$ ( $p \not \equiv$ const) найдем расстояние

$$
D(p, t)=\inf \{|t-T|: T \in \arg d(p, t)\}
$$

от точки $t$ до множества точек $T$, реализуюших минимум (4). Очевидно, что $D(p, t)$ - наилучшая точность привязки (методом $(4))$ по фрагменту $\varphi_{t}$ и функции $p$. Определим

$$
D(p)=\sup _{t}\left\{D(p, t): \Delta_{t} \subset Q\right\}
$$

В случае $p_{0} \equiv$ const $\left(p_{0} \in \mathscr{P}\right)$ положим

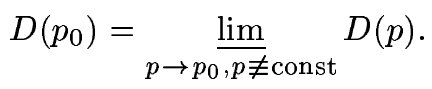


ОПРЕДЕЛЕНИЕ. Функцию $p^{*} \in \mathscr{P}$, для которой достигается нижняя грань

$$
D:=D\left(p^{*}\right)=\inf \{D(p): p \in \mathscr{P}\},
$$

назовем функиией наилучшей привязки.

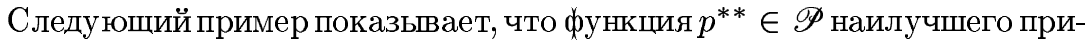
ближения в метрике $C(Q)$ для $f$ (т.е. такая, что $\left\|f-p^{* *}\right\|_{Q}=\inf \left\{\|f-p\|_{Q}\right.$ : $p \in \mathscr{P}\})$ не обязана быть функцией наилучшей привязки.

Пусть $Q=[0,1], \Delta=\{0\}, \mathscr{P}=\left\{a_{2} x^{2}+a_{1} x+a_{0}\right\}$-множество многочленов 2 -й степени, $0<\varepsilon<1 / 4, f$ - четырехзвенная кусочно-линейная функция, принимающая в точках $0, \sqrt{\varepsilon}, 1-2 \varepsilon, 1-\varepsilon, 1$ значения $\varepsilon, \sqrt{\varepsilon},(1-2 \varepsilon)^{2}-\varepsilon$, $(1-\varepsilon)^{2}+\varepsilon, 1-\varepsilon$ соответственно. Точки $\varepsilon, 1-2 \varepsilon, 1-\varepsilon, 1$ образуют для $f$ и $p=x^{2}$ чебьшевский альтернанс (см. [6]) и по теореме П. Л. Чебьшева $p-$ многочлен наилучшего приближения для $f$. Ошибка привязки с помощью $p$ равна $D(p)=D(p, 0)=\sqrt{\varepsilon}$ (при $\delta=0)$, а многочленом наилучшей привязки является $p^{*}=(x+\alpha)^{2}$, где $2 \alpha=\sqrt{\varepsilon}+2 \varepsilon+\xi-1, \xi=\sqrt{(1-2 \varepsilon)^{2}-\varepsilon}$, и при этом $D\left(p^{*}\right)=D\left(p^{*}, 0\right)=D\left(p^{*}, 1-2 \varepsilon\right)=\sqrt{\varepsilon}-\alpha<D(p)$.

ЗАмЕчАниЕ 2. Т.В. Демченко проведен вычислительньй эксперимент для функций одной и двух переменных. В качестве $\mathscr{P}$ взят класс многочленов 2-й степени, в качестве $f$-многочлены большей степени. Расчеты показали, что даже для столь гладких $f$ многочлен наилучшей привязки дает точность привязки в среднем на $7-10 \%$ выше в сравнении с многочленом наилучшего приближения.

Перечислим некоторые свойства введенных понятий [4]: отображение $(p, t) \mapsto \arg d(p, t)$ полунепрерывно сверху; функционалы $D(p, t), D(p)$ полунепрерьвны снизу; функция наилучшей привязки может быть не единственной; нижняя грань (10) может не достигаться, даже если $\mathscr{P}$ - конечномерное подпространство из $C(Q)$; функционал $D(p)$ может иметь точки локального (не глобального) экстремума.

Далее устанавливаются условия на функцию $p \in \mathscr{P}$, доставляющую функционалу $D(p)$ минимум, вообще говоря, локальный. Функция $f$ предполагается зафиксированной.

Для $p \in \mathscr{P}, t \in Q, T \in \mathbb{R}^{N}$ обозначим

$$
\begin{gathered}
r_{T}(x)=p(x+T)-f(x+t) \quad(x \in \Delta), \\
R=R(t, T)=R_{p}(t, T)=\arg \max \left\{\left|r_{T}(x)\right|: x \in \Delta\right\},
\end{gathered}
$$

$p^{\prime}(x) l=\left(p^{\prime}(x), l\right)$ - скалярное произведение векторов $p^{\prime}(x)$ и $l$, где $p^{\prime}(x)$ градиент функции $p, O_{\varepsilon}(A)=\left\{x \in \mathbb{R}^{N}: \inf _{a \in A}|x-a|<\varepsilon\right\}-\varepsilon$-окрестность $(\varepsilon>0)$ множества $A \subset \mathbb{R}^{N}$. 
ПРЕДЛОЖЕНИЕ 4. Если вектор $l \in \mathbb{R}^{N}$ удовлетворяет условию

$$
r_{T}(x) p^{\prime}(x+T) l<0 \quad \forall x \in R_{p}(t, T),
$$

то найдется $\alpha_{0}>0$, для которого

$$
\left\|r_{T+\alpha l}\right\|_{\Delta}<\left\|r_{T}\right\|_{\Delta}<\left\|r_{T-\alpha l}\right\|_{\Delta} \quad\left(0<\alpha<\alpha_{0}\right) .
$$

ДокАЗАТЕЛЬСТво. Обозначим $s=\operatorname{sign} r_{T}(x)$. Множество $R(t, T)$ компактно, а отображение $T \mapsto R(t, T)$ полунепрерывно сверху, поэтому для любого $\varepsilon>0$ существует $\delta=\delta_{\varepsilon}>0$ такое, что

$$
R(t, T+l) \subset O_{\varepsilon}(R(t, T)) \quad \forall l:|l|<\delta .
$$

В силу компактности множества $R=R(t, T)$ и непрерьвности функций $f$ и $p$ найдется $\gamma>0$ такое, что для любых $x \in R, \xi \in O_{\gamma}(x) \cap \Delta$ и любого $l,|l|<\gamma$, вьполняется условие

$$
\operatorname{sign} r_{T+l}(\xi)=\operatorname{sign} r_{T}(x),
$$

и поэтому ввиду дифференцируемости функции $p$

$$
\begin{aligned}
\left|r_{T+l}(\xi)\right|-\left|r_{T}(\xi)\right| & =s\left[r_{T+l}(\xi)-r_{T}(\xi)\right]=s[p(\xi+T+l)-p(\xi+T)] \\
& =s p^{\prime}(\xi+T) l+o(|l|) .
\end{aligned}
$$

Пусть существует $l$, удовлетворяющий условию (11). В силу компактности $R$ для некоторого $\eta=\eta_{l}<0$

$$
s p^{\prime}(x+T) l<3 \eta \quad \forall x \in R .
$$

По непрерывности $r_{T}(x)$ и $p^{\prime}(x+T)$ найдется $\varepsilon=\varepsilon_{\eta, \gamma}(0<\varepsilon<\gamma$, см. (13)) такое, что

$$
s p^{\prime}(\xi+T) l<2 \eta<0 \quad \forall \xi \in O_{\varepsilon}(R) .
$$

По $\varepsilon$ найдем $\delta=\delta_{\varepsilon, \gamma}>0(0<\delta<\gamma)$ так, чтоб выполнялось (12). Теперь выберем число $\alpha_{0}>0$ из следующих условий: $\left|o\left(\left|\alpha_{0} l\right|\right)\right|<\alpha_{0}|\eta|$ (см. (14)), $\left|\alpha_{0} l\right|<\delta_{\varepsilon}$. Для $\alpha, 0<\alpha<\alpha_{0}$, учитьвая (14), получим

$$
s r_{T+\alpha l}(\xi)-\alpha \eta<s r_{T}(\xi)<s r_{T-\alpha l}(\xi)+\alpha \eta \quad \forall \xi \in O_{\varepsilon}(R(t, T)),
$$

и ввиду (12) выполняются неравенства

$$
\left\|r_{T+\alpha l}\right\|_{\Delta}-\alpha \eta<\left\|r_{T}\right\|_{\Delta}<\left\|r_{T-\alpha l}\right\|_{\Delta}+\alpha \eta \quad\left(0<\alpha<\alpha_{0}, \quad \eta<0\right) .
$$

Предложение доказано. Из него следует теорема о необходимых условиях экстремума по $T$. 
Tеорема 1. Пусть $p \in \mathscr{P}, t \in Q, T^{*} \in \mathbb{R}^{N}, \Delta+T^{*} \subset Q u p\left(x+T^{*}\right) \not \equiv$ $f(x+t)$ на $\Delta$. Если

$$
\left\|f(x+t)-p\left(x+T^{*}\right)\right\|_{\Delta}=\min _{T}\left\{\|f(x+t)-p(x+T)\|_{\Delta}: \Delta+T \subset Q\right\},
$$

mo

$$
\bigcap_{\xi \in R\left(t, T^{*}\right)}\left\{l \in \mathbb{R}^{N}: r_{T}(\xi) p^{\prime}(\xi) l<0\right\}=\varnothing
$$

Обозначим

$$
\begin{gathered}
R^{0}=\left\{\xi \in R_{p}(t, T): p^{\prime}(\xi)=0\right\}, \\
\bar{R}=\left\{\xi \in R_{p}(t, T): r_{T}(\xi)=\left\|r_{T}\right\|_{\Delta}\right\}, \\
\underline{R}=\left\{\xi \in R_{p}(t, T): r_{T}(\xi)=-\left\|r_{T}\right\|_{\Delta}\right\}, \\
R_{l}^{ \pm}=\left\{\xi: \pm\left(p^{\prime}(\xi+T) l\right) \operatorname{sign} r_{T}(\xi)<0\right\} \quad\left(l \in \mathbb{R}^{N}\right),
\end{gathered}
$$

и для $q \in \mathscr{P}$

$$
R(q)=\arg \max \left\{q(\xi+T) \operatorname{sign} r_{T}(\xi): \xi \in R_{p}(t, T)\right\},
$$

а если $r_{T}(\xi) \equiv 0$ на $\Delta$, то

$$
R(q)=\arg \max \{|q(\xi)|: \xi \in \Delta\} .
$$

ПРЕДЛОЖЕНИЕ 5. Пусть $p \in \mathscr{P}, t \in Q, T=T_{p} \in \arg d(p, T), l \in \mathbb{R}^{N}$ $u q \in \mathscr{P}$ таковы, что

1) если $_{T}(\xi) \not \equiv 0$ на $\Delta$, то для любого $\xi \in R(q)$ либо $\left(\operatorname{sign} r_{T}(\xi)\right) \times$ $p^{\prime}(\xi+T) l<0$, либо $\left(\operatorname{sign} r_{T}(\xi)\right) p^{\prime}(\xi+T) l=0 u\left(\operatorname{sign} r_{T}(\xi)\right) \times$ $q^{\prime}(\xi+T) l<0$,

2) если $r_{T}(\xi) \equiv 0$ на $\Delta$, mо $(\operatorname{sign} q(\xi+T)) p^{\prime}(\xi+T) l<0$ для любого $\xi \in R(q)$.

Тогда найдется $\lambda_{0}>0$ maкое, что для $0<\lambda<\lambda_{0}, p_{\lambda}=p+\lambda q$ локальный минимум по $\alpha$

$$
\min \left\|p_{\lambda}(x+T+\alpha l)-f(x+t)\right\|_{\Delta}
$$

достигается при $\alpha^{*}>0$. 
ДокАЗАТЕЛЬСТво. 1) Пусть $T_{\lambda}^{l}=T+\alpha^{*} l$, где $\alpha^{*}$ - точка минимума в задаче (15). Функция

$$
\max \{|p(x+T)-f(x+T)|: x \in \Delta\}
$$

непрерьвна по $p, T$ и отображения

$$
p \mapsto \arg d(p, t), \quad p \mapsto R_{p}(t, T)
$$

полунепрерьвны сверху, поэтому для любого $\varepsilon>0$ найдется $\lambda=\lambda_{\varepsilon}>0$ такое, что при $|\lambda|<\lambda_{\varepsilon}$

$$
\left|T_{p}-T_{\lambda}^{l}\right|<\varepsilon, \quad R_{p_{\lambda}}(t, T) \subset O_{\varepsilon}\left(R_{p}(t, T)\right) .
$$

Существует $\varepsilon_{1}>0$ такое, что

$$
\operatorname{sign} r_{T}(\xi)=\left\{\begin{aligned}
1, & \xi \in O_{\varepsilon_{1}}(\bar{R}), \\
-1, & \xi \in O_{\varepsilon_{1}}(\underline{R}),
\end{aligned}\right.
$$

и, кроме того, в силу непрерьвности $p^{\prime}$ и $q^{\prime}($ см. 1))

$$
\begin{array}{ll}
p^{\prime}(\xi+T) l \operatorname{sign} r_{T}(\xi)<0, & \xi \in O_{2 \varepsilon_{1}}\left(R_{l}^{+} \cap R(q)\right), \\
q^{\prime}(\xi+T) l \operatorname{sign} r_{T}(\xi)<0, & \xi \in O_{2 \varepsilon_{1}}\left(R^{0} \cap R(q)\right) .
\end{array}
$$

Поэтому найдется $\lambda_{1}>0$ такое, что при $|\lambda|<\lambda_{1}$

$$
\left(p_{\lambda}^{\prime}(\xi+T) l\right) \operatorname{sign} r_{T}(\xi)<0, \quad \xi \in O_{\varepsilon_{1}}\left(\left(R_{l}^{+} \cup R^{0}\right) \cap R(q)\right) .
$$

В силу условия 1 ) существует $\varepsilon_{2}>0$, при котором

$$
\begin{gathered}
\min \left\{q(\xi+T) \operatorname{sign} r_{T}(\xi): \xi \in O_{\varepsilon_{2}}\left(\left(R_{l}^{+} \cup R^{0}\right) \cap R(q)\right)\right\} \\
>\max \left\{q(\xi+T) \operatorname{sign} r_{T}(\xi): \xi \in O_{\varepsilon_{2}}\left(R_{l}^{-} \cap R\right)\right\} .
\end{gathered}
$$

Для $\varepsilon=\min \left\{\varepsilon_{1}, \varepsilon_{2}\right\},|\lambda|<\min \left\{\lambda_{\varepsilon}, \lambda_{1}\right\}$ ввиду $(16) R_{p_{\lambda}}(t, T) \subset O_{\varepsilon}\left(R_{p}(t, T)\right)$, а по (18) $R_{p_{\lambda}}(t, T) \subset O_{\varepsilon}\left(\left(R_{l}^{+} \cup R^{0}\right) \cap R(q)\right)$. Отсюда, учитывая (17) и предложение 4 , получаем требуемое утверждение в случае 1$)$. В случае 2 )

$$
\operatorname{sign}\left[p_{\lambda}(x+T)-f(x+t)\right]=\operatorname{sign} q(x+T) \quad(\lambda>0),
$$

поэтому при достаточно малых $\lambda>0$

$$
\left(\operatorname{sign}\left[p_{\lambda}(x+T)-f(x+t)\right]\right) p_{\lambda}^{\prime}(x+T) l<0 \quad(x \in R(q)) .
$$

Для завершения доказательства осталось применить предложение 4. 
ЗАМЕЧАНИЕ 3 . Если $R^{0} \cap R(q)=\varnothing$, то при $r_{T}(\xi) \equiv 0$ на $\Delta$ условие 2$)$ можно записать в виде

$$
\max _{\xi \in \Delta}\left[-q(\xi+T) \operatorname{sign} p^{\prime}(\xi+T) l\right]>\max _{\xi \in \Delta} q(\xi+T) \operatorname{sign} p^{\prime}(\xi+T) l .
$$

Нетрудно привести примеры функций $p \in \mathscr{P}$ и $f$, для которых существуют $t_{k} \rightarrow t, T_{k} \rightarrow T$ такие, что $T_{k} \in \arg d\left(p, t_{k}\right)$, но $T \notin \arg d(p, t)$. Кроме того, множество $\arg D(p)$ может быть не замкнутым. В связи с этим целесообразно ввести обозначения

$$
\mathbf{t}=\mathbf{t}_{p}=\left\{t \in Q: \exists t_{k} \rightarrow t, D\left(p, t_{k}\right) \rightarrow D(p)\right\}
$$

и для $t \in Q \mathscr{T}(t)=\mathscr{T}_{p}(t)_{\Delta}$ - множество предельных точек всевозможных последовательностей $T_{k} \in \arg D\left(p, t_{k}\right)$, где $t_{k} \rightarrow t$. Множество $\mathbf{t}$ замкнуто.

Следующая теорема дает необходимые условия для функции $p \in \mathscr{P}$ наилучшей привязки.

TЕорема 2. Пусть $p \in \mathscr{P}, D(p)>0$ и отображсение $t \mapsto \mathscr{T}_{p}(t)=T(t)$ однозначно. Если р доставляет локальный минимум функиионалу $D(p)$ на классе $\mathscr{P}$, то не существует функции $q \in \mathscr{P}$ такой, что при некотором $\delta>0$ для любых $t \in \mathbf{t}$ выполняются условия:

1) для любого $l \in \mathbb{R}^{N}$, удовлетворяющего неравенству $(l, t-T) \leqslant \delta$ $(T=T(t))$ существует $\xi \in R(q)$ такой, что

a) $r_{T}(\xi)\left(p^{\prime}(\xi+T) l\right)>0$ при $r_{T}(\xi) \not \equiv 0$ на $\Delta$,

б) $q(\xi+T)\left(p^{\prime}(\xi+T) l\right)>0$ при $r_{T}(\xi) \equiv 0$ на $\Delta$;

2) существует $l \in \mathbb{R}^{N},|l|=1$, такой, что $(l, t-T)>\delta$ и для любого $\xi \in R(q)$

a) $r_{T}(\xi)\left(p^{\prime}(\xi+T) l\right)<0$ nри $r_{T}(\xi) \not \equiv 0$ на $\Delta$,

б) $q(\xi+T)\left(p^{\prime}(\xi+T) l\right)<0$ при $r_{T}(\xi) \equiv 0$ на $\Delta$.

ДокАЗАТЕЛЬСтво. Предположим, что существует $q \in \mathscr{P}$, удовлетворяющая указанным свойствам. Ввиду полунепрерывности сверху отображения $(p, t) \mapsto \arg d(p, t)$ и компактности множества $\mathbf{t}$ для любого $\varepsilon>0$ найдется $\lambda_{\varepsilon}>0$ такое, что для каждого $t \in \mathbf{t}$ любая точка $T_{\lambda}=T_{\lambda}(t)$ минимума функционала

$$
\left\|p_{\lambda}(x+T)-f(x+t)\right\|_{\Delta} \quad\left(0 \leqslant \lambda<\lambda_{\varepsilon}\right)
$$

удовлетворяет неравенству $\left|T_{\lambda}(t)-T(t)\right|<\varepsilon$. Выберем $\varepsilon$ настолько малым, что неравенства 1a), 1б) выполняются в $\varepsilon$-окрестностях точек $\xi$, а 
неравенства 2а), 2б) - в $\varepsilon$-окрестности множества $R(q)$ и, кроме того, множество

$$
K_{\varepsilon, \delta}(t)=\{T+\alpha \cdot l:|l|=1,0<\alpha \leqslant \varepsilon,(l, t-T) \geqslant \delta\}
$$

содержится внутри шара радиуса $D(p)$ с центром в точке $t$. Из условий 1$), 2)$ теоремы и предложения 5 следует, что $T_{\lambda} \in K_{\varepsilon, \delta}(t)$ и, значит, $\left|t-T_{\lambda}\right|<|t-T|$. Ввиду непрерьвности $p^{\prime}(\xi)$ ограничен на $\mathbf{t}$, поэтому

$$
\inf \left\{|t-T|-\left|t-T_{\lambda}\right|: t \in \mathbf{t}\right\}>0 \text {. }
$$

Отсюда следует, что $p$ не является “точкой” минимума функционала $D(p)$.

Приведенная теорема является основой численных алгоритмов построения функции $p \in \mathscr{P}$ наилучшей привязки. "Полином" $q \in \mathscr{P}$, удовлетворяющий условиям 1), 2) теоремы, задает направление спуска для функционала $D$, т.е. $D(p+\lambda q)<D(p)$ для достаточно малых $\lambda>0$.

Автор благодарит Ю. Н. Субботина за полезные обсуждения.

Институт математики и механики

Поступило

Уральского отделения РАН

26.08 .94

E-mail: bvi@apprekat.imm.intec.ru

Исправленный вариант

23.05 .95

\section{СПИСОК ЦИТИРОВАННОЙ ЛИТЕРАТУРЫ}

[1] Белоглазов И. Н., Джанжгава Г. И., Чигин Г. П. Основы навигации по геофизическим полям. М.: Наука, 1985.

[2] Красовский А.А., Белоглазов И.Н., Чигин Г. П. Теория корреляционно-экстремальных навигационных систем. М.: Наука, 1979.

[3] Бердышев В. И. Полиномиальная аппроксимация, связанная с навигацией по геофизическим полям // Докл. РАН. 1992. Т. 6. С. 1099-1102.

[4] Berdyshev V. I. Polynomial approximation and geophysical-field navigation problem // Russian J. Numer. Anal. Math. Modelling. 1993. V. 8. № 2. P. 83-100.

[5] Никольский С. M. Приближение функций многих переменных и теоремы вложения. М.: Наука, 1969.

[6] Натансон И. П. Конструктивная теория функций. М.-Л.: ГИТТЛ, 1949. 